\title{
Exploration of anti-Malassezia potential of Nyctanthes arbor-tristis L. and their application to combat the infection caused by Mala s1 a novel allergen
}

Rohit K. Mishra ${ }^{1 *}$, Vani Mishra ${ }^{2 *}$, Anand Pandey ${ }^{3}$, Amit K. Tiwari $^{3}$, Himanshu Pandey ${ }^{4}$, Shivesh Sharma', Avinash C. Pandey ${ }^{2}$ and Anupam Dikshit ${ }^{3^{*}}$

\begin{abstract}
Background: Malassezia commensal yeasts along with multitude of antigens have been found to be associated with various skin disorders including Pityriasis versicolor (PV). Amongst them Mala s1, a 37 kDa protein has been proved to be a major allergen reacting with a large panel of sera. However, there exists no therapeutic alternative to combat such problems in form of plant based natural compounds. The purpose of this study is in the first place, to determine the anti-Malassezia activity of Nyctanthes arbor-tristis L. (NAT) ethanolic leaf extract through turbidimetric growth curves, disruption of plasma membrane and secondly, it aims to present in silico validation of its active constituents over Mala s1a novel allergen.
\end{abstract}

Methods: The antifungal susceptibility $50 \%$ ethanolic extract of NAT was determined by broth microdilution method according to CLSI guidelines. Further MICs and IC 50 were determined spectrophotometrically using the software SoftMax ${ }^{\circledast}$ Pro-5 (Molecular Devices, USA). Active constituents mediated disruption of plasma membrane was studied through flowcytometry by permeabilization of fluorescent dye Propidium lodide (PI). Antioxidant activity of the extract was determined using the DPPH stable radical. Molecular validation of fungal DNA from the extract was observed using PCR amplification. In silico analysis of its active constituents over Mala s1 was performed using HEX software and visualized through Pymol.

Results: The anti-Malassezia potential of NAT leaf extracts reflected moderate MIC $1.05 \mu \mathrm{g} / \mu$ lagainst M. globosa, while least effective against $M$. restricta with MIC $1.47 \mu \mathrm{g} / \mu \mathrm{l}$. A linear correlation coefficient $R^{2}=0.866$ was obtained in case of M. globosa while minimum was observed in $M$. restricta with $R^{2}=0.732$. The flow cytometric data reveal $\sim 75 \%$ cell death when treated with active constituents $\beta$-Sitosterol and Calceolarioside A. The docking confirmations and the interaction energies between Mala s1 and the active constituents ( $\beta$-Sitosterol and Calceolarioside A) from extracts showed an effective binding which suggests Mala s1 as efficient allergen for site specific targeting.

(Continued on next page)

\footnotetext{
* Correspondence: rohit_ernet@yahoo.co.in; vanimish@gmail.com; anupambplau@rediffmail.com

${ }^{1}$ Centre for Medical Diagnostic and Research (CMDR), Motilal Nehru National Institute of Technology (MNNIT), Allahabad 211004, India

${ }^{2}$ Nanotechnology Application Centre (NAC), University of Allahabad,

Allahabad 211002, India

${ }^{3}$ Department of Botany, Biological Product Lab., University of Allahabad,

Allahabad 211002, India

Full list of author information is available at the end of the article
}

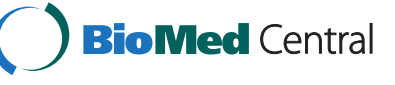

(c) 2016 Mishra et al. Open Access This article is distributed under the terms of the Creative Commons Attribution 4.0 International License (http://creativecommons.org/licenses/by/4.0/), which permits unrestricted use, distribution, and reproduction in any medium, provided you give appropriate credit to the original author(s) and the source, provide a link to the Creative Commons license, and indicate if changes were made. The Creative Commons Public Domain Dedication waiver (http://creativecommons.org/publicdomain/zero/1.0/) applies to the data made available in this article, unless otherwise stated. 
(Continued from previous page)

Conclusions: This study revealed that Nyctanthes arbor-tristis L. (NAT) extracts possess high anti-Malassezia potential which is driven mainly by disruption of plasma membrane. Also in silico validation and molecular modeling studies establishes Mala s1 as a novel allergen that could be a potential target in disease treatment. Our results would also provide a foundation for the development of new therapeutic approach using NAT extract as lead compound with high antioxidant property as an added trait for skin care.

Keywords: Nyctanthes arbor-tristis L. (NAT), Anti-Malassezia susceptibility, AUGC, Flow cytometry, Mala s1, Molecular docking

\section{Background}

Malassezia species (formerly Pityrosporum ovale) are exclusively the single eukaryotic member that forms a part of the resident skin microflora of human and other warm-blooded vertebrates with wide intraspecies diversity $[1,2]$. This intra-species diversity could be attributed to fungal evolution due to ecology, host adaptation, and pathogenicity [3]. These are opportunistic yeasts requiring predisposing environmental circumstances such as temperature and humidity, patient immune status, and genetic susceptibility [4]. Malassezia spp. are associated with different superficial pathogenesis, including Pityriasis versicolor (PV), Seborrheic dermatitis (SD), folliculitis as well as nosocomial bloodstream infection in pediatric care units $[5,6]$. They have a species-specific ability to interact with cells and structures associated with skin, e.g. various keratinocyte subpopulations. Cell lineages involved in immune functions, including Antigen-presenting Dendritic Cells, Pattern Recognition Receptors (PRRs), Macrophages, Eosinophils and Neutrophils have also been found to be affected by Malassezia spp. [7, 8]. Moreover, the host interaction with Malassezia yeast can stimulate sensitization; elicit IgE production and T-cell reactivity. Malassezia extract contains a wide range of IgE binding proteins [9], however, variations in allergenic content exist. Experimental findings establish Mala s1 as a major allergen in skin disorders with $58 \%$ IgE binding frequency [10, 11]. Mala s1 is of particular importance since it is localized in the cell wall and therefore, is easily accessible for the interplay with human innate and acquired immune system.

Many azoles derivatives are frequently used as preventive measures against fungal infections [12, 13]. However, in response to synthetic antifungal, development of resistant strains [14] and recapitulation of the disease symptoms with severe side effects have important implications in health care [15]. According to Jesus et al. [16] Malassezia spp. was found to be susceptible to various azole derivatives with MICs ranging from 0.01 to $4 \mu \mathrm{g} / \mathrm{ml}$. However, isolates generated through in-vitro induction of resistance against fluconazole exhibited increased MICs ranging from 64 to $128 \mu \mathrm{g} / \mathrm{ml}$. These strains were also found to be resistant against other azole derivatives. In recent years, natural products have been used in the discovery and development of drugs [17]. Complementary and Alternative medicines (CAM) approaches and interventions have been found to exert their effect in boosting immune response and reducing pathogen-associated symptoms [18]. In recent years they have become important molecular tools for identification of various cellular interactions because they are capable of binding specific target proteins and then interfere with their metabolism.

In India, hundreds of botanicals have traditionally been used as therapeutic alternative for majority of diseases. Many of these plants are commonly used even today. In support of this fact recently, we reported ethanolic extract of lichen Cladia to be effective against Malassezia spp. [19]. Nyctanthes arbor-tristis L. (NAT) commonly known as Night Jasmine (Parijatha) is one amongst them. The decoction of leaves is extensively used by Ayurvedic physicians for the treatment of arthritis, obstinate sciatica, malaria, intestinal worms and as a tonic, cholagogue and laxative [20]. In addition, analgesics, antipyretic along with ulcerogenic potency have also been observed [21]. The plant extract has also been defined to possess potent immunomodulatory activity through strong stimulation of antigen-specific and non-specific immunity as shown by increased humoral and delayed type hypersensitivity responses [22]. A water-soluble fraction of the ethanol extract elicits significant antiinflammatory activity against acute inflammatory oedema produced in in-vivo models [23]. The $50 \%$ ethanolic seed and root extracts of NAT also show immunomodulatory activity in systemic candidiasis infections in mice [24]. Active constituents like calceolarioside $\mathrm{A}$ and $\beta$-sitosterol from leaves have been reported as an efficient anti-inflammatory, antileishmanial and anticancer compound [25]. Thus, considering the evidence of antimicrobial and immunomodulatory potential, it would be interesting to study if the plant ethanolic extract is somehow involved in the modulation of infection caused by Malassezia. Anti-Malassezia susceptibility was studied 
using CLSI broth microdilution method which has recently been accepted widely [26]. Moreover, the interaction of Malassezia yeast with the skin results in exhaustive damage to skin baarier and its structures [27]. The dermal reconstruction is attributed to efficient synchronization of skin cells and immunomodulatory factors [28]. Accumulated research indicates effective role of antioxidant potency of botanicals in treating degenerative diseases [29, 30]. Thus, in order to address the problem of skin damage caused in PV, ethanolic NAT extract has also been assessed for its anti oxidant capacity through DPPH (1,1-Diphenyl-2-picryl-hydrazyl) assay.

Interestingly, Mala s1 has been spotted as the major allergen in the yeast and its capacity to stimulate the immune system is well documented, but its antigenicity has not yet been elucidated. Despite the fact that its 3D structure and localization in the cell wall have been elaborated, their functional details are still an enigma. Therefore, in the current study, we took a top-down approach to identify the antifungal potential of NAT leaf extract through broth microdilution assay and PCR. We systematically monitored the growth patterns of the yeast to establish the antifungal susceptibility of the plant extract. We also studied whether plant extract and the active constituents could bring about changes in cell membrane integrity by measuring Propidium Iodide (PI) influx through flow cytometer. Taking advantage of exclusive prior knowledge of NAT and its active constituents, we systematically screened two molecules by virtue of their antimicrobial ability ( $\beta$-Sitosterol and Calceolarioside A) and predicted their binding on Mala s1 through molecular simulations.

\section{Methods}

\section{Plant material}

The fresh leaves of Nyctanthes arbor-tristis L. (NAT) were collected from Roxburgh garden, University of Allahabad, India. The plant was identified and authenticated from Botanical Survey of India (BSI), Allahabad and voucher specimen was kept in Duthie Herbarium, Dept. of Botany, University of Allahabad with reference number BPL/NAT/1009.

\section{Microorganisms}

Four strains $M$. furfur 1878; M. restricta 7877; M. globosa 7966 and M. sympodialis 9974 were procured from Central Bureau voor Schimmelcultures (CBS) Fungal Biodiversity Centre, Institute of the Royal Netherlands Academy of Arts and Sciences (KNAW), Netherlands (Fig. 1).

\section{Preparation of ethanolic extract}

Freshly collected leaves of Nyctanthes arbor-tristis L. (NAT) were cut into pieces and shade dried at room temperature. They were then minced using mortar. The processed sample was mixed in $50 \%$ ethanol (1:5) for $24 \mathrm{~h}$. The extracts were finally filtered and concentrated using Rotatory evaporator (Buchi type) and stored at $4{ }^{\circ} \mathrm{C}$.

\section{Antifungal susceptibility test}

The antifungal activity of $50 \%$ ethanolic extract of NAT was determined using broth microdilution in 96-well flat bottom microtiter plates according to CLSI guidelines [31, 32]. Briefly, ethanolic extract of NAT was dissolved in 5-10\% DMSO to obtain a stock solution of $50 \mu \mathrm{g} / \mu \mathrm{l}$, which was then serially diluted 1:10 in medium in order to attain final

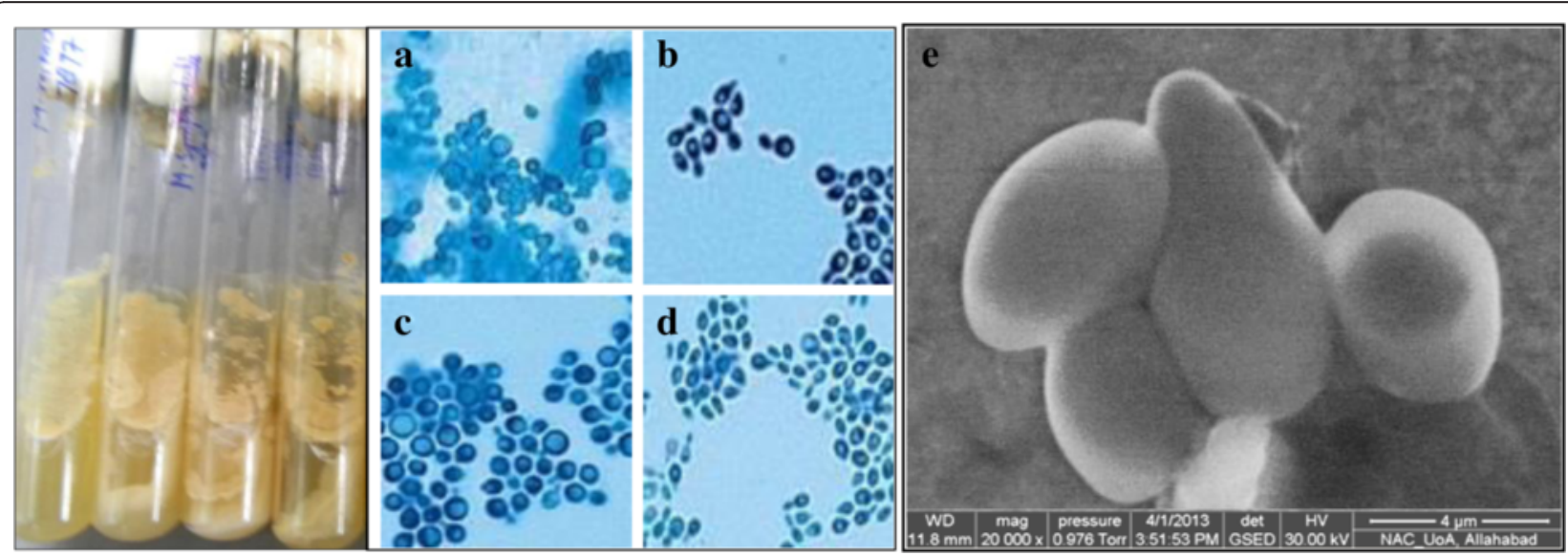

Fig. 1 Standard strains of Malassezia sp. a M. furfur b M. restricta c M. globosa d M. sympodialis. Cultures were procured from Royal Netherlands Academy (Netherland) and grown in Modified Leming and Notman Agar (MLNA) and were visualized microscopically (a to d) e Scanning electron micrograph (SEM) of Mala s1 allergen producing Malassezia sympodialis (Magnification 20,000X, Pressure 0.976 Torr, WD 11.8 mm) 
concentration ranging from 0.02 to $2.5 \mu \mathrm{g} / \mu \mathrm{l}$. Each well was subsequently filled with $100 \mu \mathrm{l}$ of inoculum. The initial concentration of inoculum was $1 \times 10^{6} \mathrm{cell} / \mathrm{ml}$ (adjusted according to $0.5 \mathrm{McFarland)}$ in liquid media BPL5O (Patent application no. DEL/546/2012) [33, 34]. The plates were incubated at $35 \pm 2{ }^{\circ} \mathrm{C}$ in wet chamber for $72 \mathrm{~h}$ and experiments were conducted in triplicate.

\section{Quantification of fungal growth}

After $12 \mathrm{~h}$ of incubation, the optical density of the microtiter plates was recorded spectrophotometrically at $530 \mathrm{~nm}$ using SpectraMaxplus ${ }^{384}$ (Molecular Devices, USA). The process was repeated after every $12 \mathrm{~h}$ of incubation and continued till $72 \mathrm{~h}$. The changes in OD over time were used to generate growth curves at each drug concentration against the control. The normalized OD of the extract treated wells (OD obtained after subtraction of the background OD) was used for the generation of turbidimetric growth curves. Percentage of growth inhibition at each drug concentration was calculated using the formula:

$$
\% \text { growth }=\frac{\mathrm{OD}_{530} \text { of wells containing the extract }}{\mathrm{OD}_{530} \text { of the extract free well }} \times 100
$$

\section{Area under growth curve (AUGC) analysis}

AUGC was calculated and integrated using SoftMax ${ }^{\oplus}$ Pro-5 (Molecular Devices, USA). Potential differences in the $\mathrm{OD}_{530}$ among individual wells were accounted for by subtracting the initial $\mathrm{OD}_{530}$ from the values measured during the $72 \mathrm{~h}$ incubation, yielding net $\mathrm{OD}_{530}$ changes for each well. The curve was generated between AUGC vs NAT leaf extract concentration (log transformed) and a linear regression analysis was performed in MS Excel.

Determination of minimum inhibitory concentrations (MICs) MICs were determined spectrophotometrically using the software SoftMax ${ }^{\ominus}$ Pro-5 (Molecular Devices, USA). For the NAT extract, the MIC was determined as the lowest drug concentration showing $\geq 80 \%$ growth inhibition compared with the growth in the extract-free well. Each test was performed for triplicates.

\section{Determination of minimum fungicidal concentration (MFC)}

MFC was defined as the lowest concentration of the plant extract at which $99.99 \%$ or more of the initial inoculum was killed. $100 \mu \mathrm{l}$ aliquot of inoculum was taken aseptically from those wells that did not show turbidity and was poured on Modified Leming and Notman Agar (MLNA) agar plates followed by incubation for 4 days at $35 \pm 2{ }^{\circ} \mathrm{C}$. Absence of growth reflected that the concentration was cidal. The number of surviving organisms was determined by viability counts. All tests were performed for triplicates.

\section{Molecular validation of fungal DNA from the extract using PCR amplification}

Cells from the wells were collected for DNA extraction using AccuPrep ${ }^{\circ}$ Genomic DNA Extraction Kit (Bioneer, South Korea) according to the manufacturer's protocol. PCR was performed using 4X Universal Hot Start PCR Premix (Professional Biotech India Ltd., India). The thermal cycler conditions followed were: Initial PCR activation at $95{ }^{\circ} \mathrm{C}$ for $15 \mathrm{~min}$ followed by 45 cycles of denaturation at $94{ }^{\circ} \mathrm{C}$ for $15 \mathrm{~s}$, annealing at a $5{ }^{\circ} \mathrm{C}$ below the Tm of primers for $30 \mathrm{~s}$ and extension of $30 \mathrm{~s}$ at $72{ }^{\circ} \mathrm{C}$ and final extension at $72{ }^{\circ} \mathrm{C}$ for $10 \mathrm{~min}$. Primers were designed from 26S rDNA and ITS regions of all four Malassezia species. The primer sequences were (F) 5' ATCCTTTGCAGACGACTTGA 3' and (R) 5'TGCTT AACTTCGCAGATCGG 3'. PCR products were run on $1.2 \%$ agarose gel containing ethidium bromide and then viewed on Gel Documentation system (Vilber Lourmate, France) and analyzed using VISION-CAPT software (v14.3). Molecular weight marker $(0.5 \mu \mathrm{g})$ containing a defined quantity of each band was loaded.

\section{Flow cytometric analysis for plasma membrane permeabilization}

The membrane permeability of fungal cells after treatment with the active constituents (Calceolarioside and $\beta$ Sitosterol) was measured by Propidium Iodide (PI) influx through flow cytometer. The two active constituent's $\beta$ Sitosterol (Product No. S1 270, CAS No. 83-46-5) and Calceolarioside A (Product No. SMB00246, CAS No. 84744-28-5) were procured from Sigma-Aldrich. Cells were incubated with the two compounds at concentrations 0.31 and $0.62 \mu \mathrm{g} / \mu \mathrm{l}$ for $12 \mathrm{~h}$ at $35 \pm 2{ }^{\circ} \mathrm{C}$. Following incubation, cells were washed and resuspended in PBS and subsequently stained with PI $(30 \mu \mathrm{g} / \mathrm{ml})$ for $20 \mathrm{~min}$. The cells were analyzed by BD Accuri C6 (Becton Dikinson, San Jose, CA, USA). Intrinsic parameter (SSCA) and fluorescence in FL-2 channel for PI were acquired and recorded over logarithmic scale. The changes in treated cells were compared with untreated cells as well as with cells treated with whole NAT extract.

Diphenylpicryl-hydrazyl (DPPH) radical scavenging capacity The antioxidant activity of the extract was determined in terms of hydrogen donating or radical scavenging ability using the DPPH stable radical. The assay was performed according to Brad-Willam et al. [35] with slight modifications. DPPH cation solution was prepared by adding $400 \mu \mathrm{M}$ DPPH in $0.2 \mathrm{M}$ MES buffer $(\mathrm{pH} 6)$ and $20 \%(\nu / v)$ Ethanol in equal volumes. $10 \mu \mathrm{g} / \mu \mathrm{l}$ of plant extract was prepared in 5-10\% of DMSO. The stock solution was 
further diluted to $2.5 \mu \mathrm{g} / \mu \mathrm{l}$ in 96 well Microtitre plate. DPPH cation solution was pipetted in each well and mixed with plant extract (3:1) and incubated in dark at room temperature for $20 \mathrm{~min}$. Absorbance was recorded spectrophotometrically by SpectraMaxplus ${ }^{384}$ at $517 \mathrm{~nm}$. Radical scavenging activity of the samples was expressed as $\mathrm{IC}_{50}$ which is the concentration of the sample required to inhibit $50 \%$ of DPPH concentration. Ascorbic acid was taken as standard anti-oxidant with $\mathrm{IC}_{50} 0.5 \mu \mathrm{g} / \mu \mathrm{l}$. The percent inhibition was taken into account by its FRSA count and calculated by the following formula:

$$
\% \text { Inhibition }=\frac{\text { O.D. blank-O.D. sample }}{\text { O.D. sample }} \times 100
$$

\section{In-silico analysis and molecular modeling}

$\beta$-Sitosterol and Calceolarioside A were obtained from Pubchem of NCBI (http://pubchem.ncbi.nlm.nih.gov/) in SD (structural data) format and converted to 3D structure using Pc3D Molecular Viewer (Fig. 2).

Crystal structure of Mala s1 allergen of Malassezia sympodialis (PDB id-2P9W) was retrieved from Protein Data Bank (www.rcsb.org). The active binding sites of the allergen were determined using Q-site identifier [36]. Around 10 sites were identified by analysing clusters of energetically favorable methyl binding sites to predict the ligand binding sites [37]. The PDB structures after molecular dynamics and simulations were submitted to HEX server [38]. The parameters used for the docking process were correlation type-shape only, calculation device- GPU, number of solutions-100, FFT mode -3D fast lite, grid dimension-0.6, receptor range-180, ligand range180, twist range-360, distance range-40. HEX works on FFT correlation using spherical polar coordinates and Gaussian density representation of protein shape. The results were compared on the basis of docking energy and the structures were visualized through Pymol (www.pymol.org).

\section{Statistical analysis}

All experiments were independently repeated in triplicates. Results were expressed as mean \pm Standard error (SE). Linear Regression analysis was performed to determine the correlation between AUGC and drug concentration of different Malassezia spp. The statistical analysis was performed in MS Excel.

\section{Results}

In-vitro antifungal susceptibility using turbidimetric growth analysis

The growth curves generated spectrophotometrically revealed variation in shape as well as growth rate with respect to extract concentrations, however, a consistent pattern was observed among the replicates. AUGC, as an important marker for pharmaco-dynamic study of invitro antifungal susceptibility, was also studied. Growth curves of $M$. furfur, M. restricta, $M$. globosa and $M$. sympodialis represented for $0-72 \mathrm{~h}$ at different concentrations of NAT extract are shown in Figs. 3a-i, b-i and 4a-i, b-i. Although, each growth phase could have been affected by each concentration in various ways (viz., cell wall or membrane damage, signaling blockage damage etc.), the most obvious change reflected was the shift of the growth curve towards right in response to the increasing concentration of the extract. Experiments were performed with four fungal strains over a similar dose range and conditions. Since, the results represent an undeviating pattern for all tested strains, the first result set of $M$. furfur was discussed and analyzed. The $t_{\text {lag }}$ phase in all the growth curves stretched till $24 \mathrm{~h}$ that exhibited no specific changes in OD as well as AUGC values that were lower than $2 \%$ of the maximal (data not shown) at all concentrations. However, significant changes were observed during the exponential growth phase of fungus. The control represented rapid increase in OD and about $76 \%$ increase in AUGC was reached. The extract concentration ranging from 0.02 to $2.5 \mu \mathrm{g} / \mu \mathrm{l}$ exhibited a down regulation of OD values, thereby reducing the AUGC from $52 \%$ for $0.02 \mu \mathrm{g} / \mu \mathrm{l}$ to $28 \%$ for $1.25 \mu \mathrm{g} / \mu \mathrm{l}$. The stationary phase recorded no prominent changes in OD and AUGC, thereby indicating fungal growth inhibition.

\section{In-vitro antifungal susceptibility for determination of MICs and MFCs}

The extract showed moderate MIC of $1.05 \mu \mathrm{g} / \mu \mathrm{l}$ against $M$. globosa, while least effective against $M$. restricta with their MIC of $1.47 \mu \mathrm{g} / \mu \mathrm{l}$ (Table 1). However, ethanolic
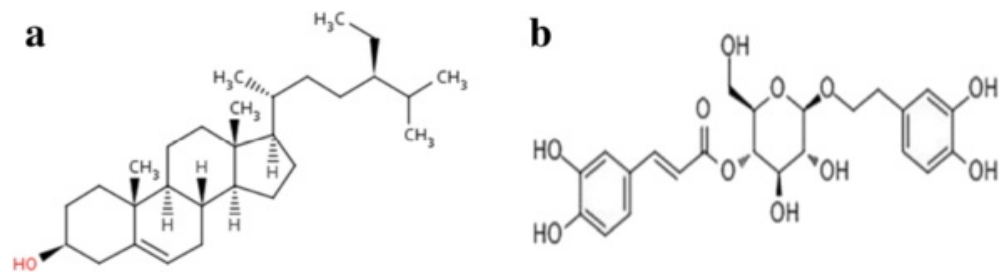

Fig. 2 Chemical structures a $\beta$-Sitosterol (Pubchem ID-222284); b Calceolarioside A (Pubchem ID-5273566) 



Fig. 3 Graphical representation of the growth of a M. furfur-1878 and b M. restricta-7877 against NAT leaf extract concentrations of 0.02 (- - ) ,

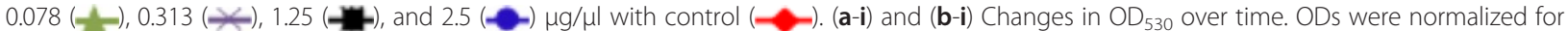
each growth curve by subtracting the background OD at each time point. (a-ii) and (b-ii) \% growth inhibition over concentration. Data represent mean \pm standard error of three replicates and * represents MIC
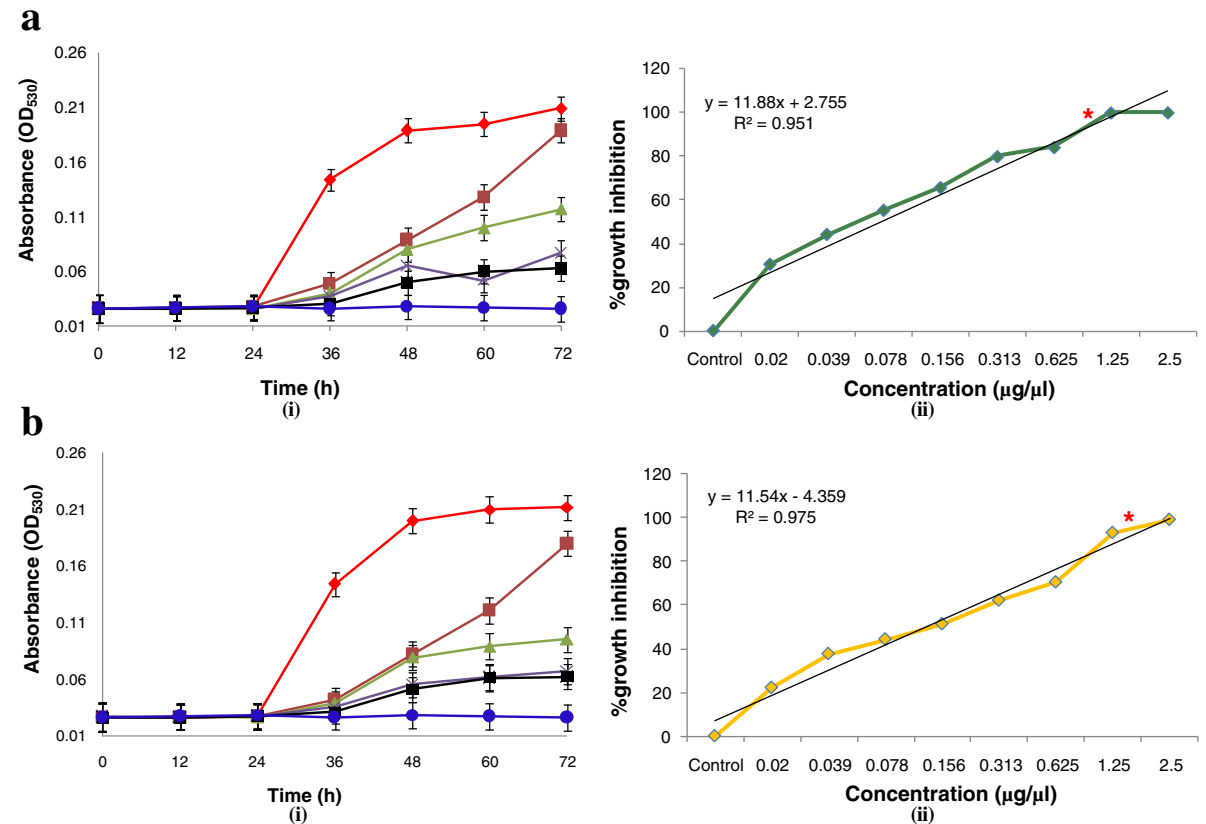

Fig. 4 Graphical representation of the growth of a M. globosa-7966 and b M. sympodialis-9974 against NAT leaf extract concentrations of 0.02

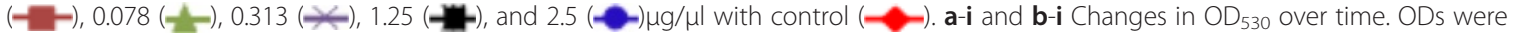
normalized for each growth curve by subtracting the background OD at each time point. a-ii and $\mathbf{b}$-ii $\%$ growth inhibition over concentration. Data represent mean \pm standard error of three replicates and * represents MIC 
Table 1 Antifungal susceptibility parameters of NAT leaf extract against Malassezia spp. $(\mu \mathrm{g} / \mu \mathrm{l})$

\begin{tabular}{lllll}
\hline \multirow{2}{*}{$\begin{array}{l}\text { Antifungal } \\
\text { parameters }\end{array}$} & $\begin{array}{l}\text { Pityriasis versicolor (PV) causing Malassezia strains procured } \\
\text { from CBS, Netherlands }\end{array}$ \\
\cline { 2 - 5 } & M. furfur & M. restricta & M. globosa & M. sympodialis \\
& 1878 & 7877 & 7966 & 9974 \\
\hline MIC & 1.22 & 1.47 & 1.05 & 1.35 \\
IC $_{50}$ & 1.17 & 1.11 & 0.74 & 1.31 \\
MFC & 3.12 & 3.12 & 3.12 & 3.12 \\
\hline
\end{tabular}

NAT extract exhibited MFC at a higher concentration $(3.12 \mu \mathrm{g} / \mu \mathrm{l})$ against all four tested strains (Fig. 1). The percent growth inhibitions of all four tested strains are expressed as Figs. 3a-ii, b-ii and 4a-ii, b-ii. A curve was plotted between extract concentration and percent growth inhibition. The graphic was complemented with a linear regression coefficient to analyze correlation between extract concentrations and percent inhibition. Significant correlation was found between extract concentrations and percent growth inhibition with a maximum $R^{2}=0.975$ for $M$. restricta. Moreover, similar correlations were observed with other groups of tested strains suggesting that the data may be useful for further primary modeling of antifungal extract.

The linear relationship resulted when the AUGC data were plotted as a function of antifungal extract (both log transformed) concentrations over the working range of the assay $(0.02$ to $2.5 \mu \mathrm{g} / \mu \mathrm{l})$. The correlation coefficient between the AUGC and concentration of antifungal extract is expressed in Fig. 5. A maximum correlation coefficient was observed for M. globosa with $R^{2}=0.866$ and minimum was observed in case of $M$. restricta with $R^{2}=$ 0.732 . Intermediate values were observed for $M$. sympodialis $\left(R^{2}=0.804\right)$ and $M$. furfur $\left(R^{2}=0.837\right)$. AUGC was highly correlated with the applied concentrations of

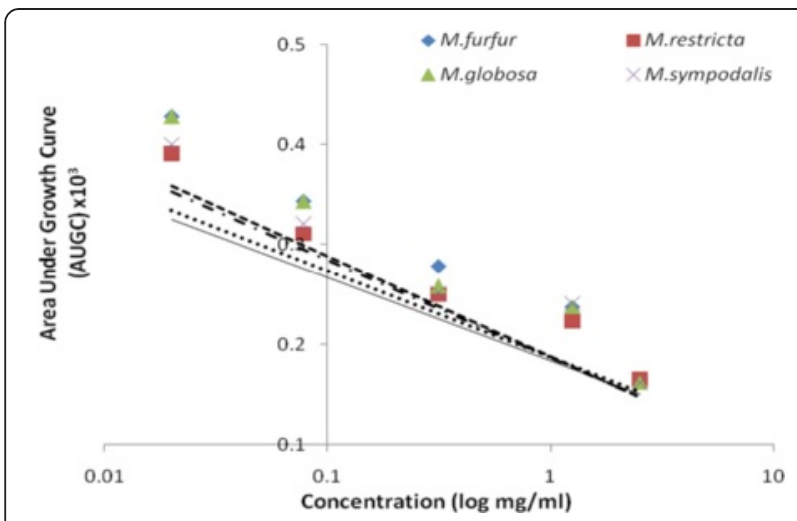

Fig. 5 Representative linear regression (AUGC) curves for growth of Malassezia furfur (- short dash; $\left.R^{2}=0.837\right)$, M. restricta $($ solid line; $\left.R^{2}=0.732\right)$, M. globosa ( $\left(\right.$ long dash; $\left.R^{2}=0.866\right)$ and $M$. sympodialis $\left(\cdots \cdots\right.$ point dash; $\left.R^{2}=0.804\right)$ as functions of NAT leaf extract concentration (log transformed) extract and was found to decrease significantly with the increasing concentration. An overlapping correlation pattern was observed for all the tested strains that converged at the same point indicating that MIC values fall under the same concentration range.

\section{Molecular validation of fungal DNA treated with extract using PCR amplification}

The preliminary evidence of antifungal susceptibility was further validated by PCR amplification. The gel image revealed low band intensities of amplicons in treated DNA samples against comparison to the control samples. In the present study, results, therefore, reflect a good relationship between MIC and the DNA expression, suggesting that NAT extract could deplete fungal DNA thus restricting fungal growth (Fig. 6).

\section{Impact of active constituents on plasma membrane lesion using flowcytometry}

To evaluate the effect of active constituents over plasma membrane, we measure the PI influx in cells was measured by flow cytometer. Both $\beta$-Sitosterol and Calceolarioside A treatment has shown an increase in PI influx. Figure $7 \mathrm{a}$ reveals no internalization of PI that represents cells with intact plasma membrane. However, a dose dependent response has been observed in active constituent treated cells. $\sim 42.2$ and $74.3 \%$ cells have been found to show PI internalization due to lesions in plasma membrane when treated with 0.31 and $0.62 \mu \mathrm{g} / \mu \mathrm{l}$ of $\beta$-Sitosterol (Fig. 7c, d). Furthermore, similar concentrations of Calceolarioside A resulted in $\sim 60.1$ and $83.2 \%$ cell death respectively (Fig. 7e, f). The whole NAT extract also showed $\sim 75.1 \%$ cell death but with a higher dose concentration that corresponds to the MIC value $(1.31 \mu \mathrm{g} / \mu \mathrm{l})$ (Fig. 7b).

\section{Antioxidant activity}

The leaf extract of NAT showed good antioxidant activity using Diphenylpicryl-hydrazyl (DPPH) radical scavenging capacity, which was clearly reflected in the results showing $\mathrm{IC}_{50}$ of $2.5 \mu \mathrm{g} / \mu \mathrm{l}$. The reference standard used was Ascorbic acid with $\mathrm{IC}_{50} 10 \mu \mathrm{g} / \mathrm{ml}$. The efficiency of plant leaf extract as potential antioxidant is an added trait (Fig. 8).

In-silico molecular simulation through molecular modeling In order to understand the inhibition mechanism of Malassezia sp. through Mala s1 allergen by natural compounds primary docking calculations were performed. The aim of the initial docking was to generate as many near native complex structures (hits) as possible. About 10 active binding sites were identified based on the interaction energy between the protein and a simple van der Waals probe. Figure 9a represents the structure and binding site of Mala s1 allergen, while Fig. 9b is the 


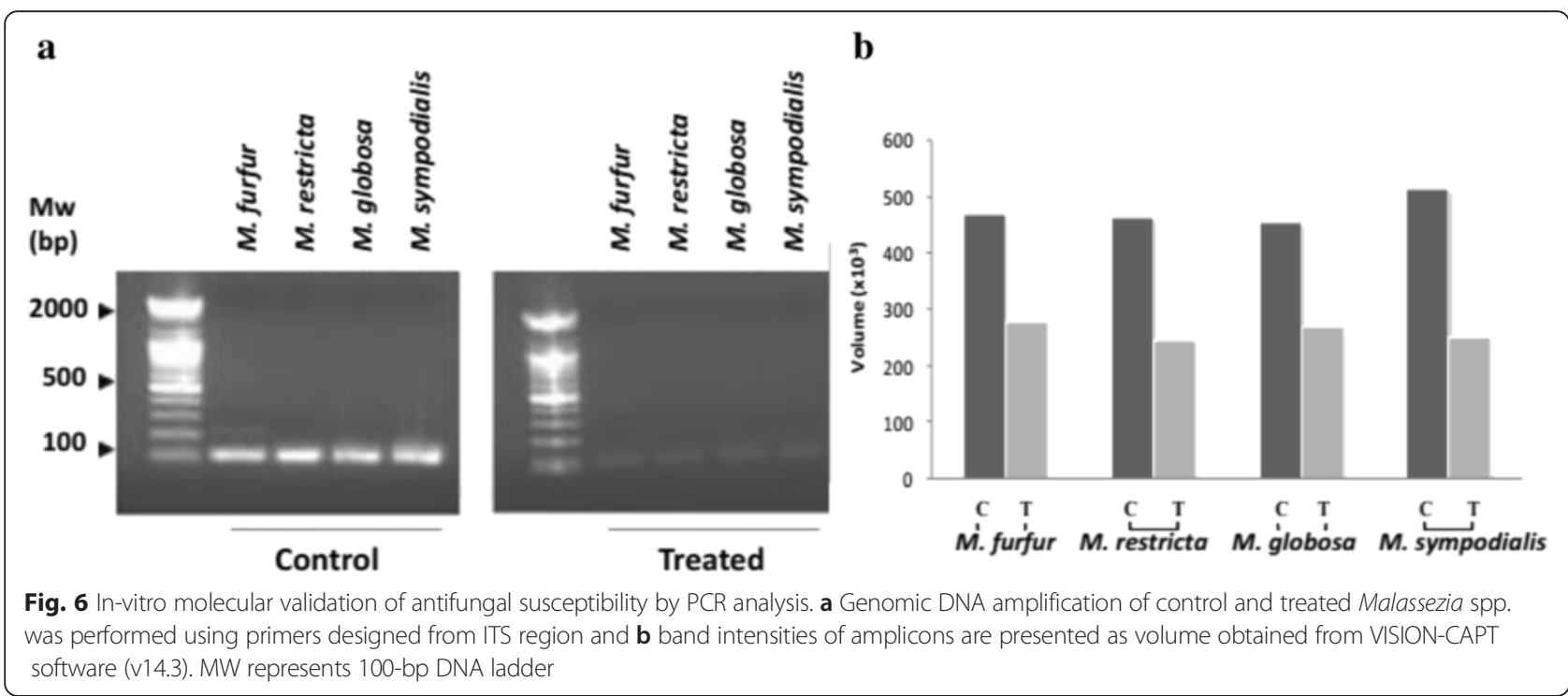

cartoon representation of the protein with Site 1 as the most favorable binding site (obtained from software server). The volume of Site 1 was estimated to be 685cubic $\AA$ and it comprises of 11 residues namely Asp39, Thr40, Ile41, Tyr42, Leu90, Ser91, Leu92, Leu93, Thr94, and His95. Following molecular dynamics simulation, the protein Mala s1 allergen with its binding site in PDB format was loaded to HEX as receptor, while the natural active constitutes found in NAT leaf extract ( $\beta$ Sitosterol and Calceolarioside A) were loaded as ligands separately. Docking was performed to analyze their binding conformations. Analysis was based on Etotal or free energy of binding. For each ligand, number of hits and total energy calculations, based on shape complementarity, are listed in Table 2 for $\beta$-Sitosterol and Table 3 for Calceolarioside A.

The docking conformation of Cluster 1 of protein with $\beta$-Sitosterol showed Etotal and RMSD values of 283.1 $\mathrm{Kcal} / \mathrm{mol}$ and $-1 \mathrm{Kcal} / \mathrm{mol}$. Similarly, Cluster 1 of Protein-Calceolarioside rated Etotal of $354.9 \mathrm{Kcal} / \mathrm{mol}$ and RMSD of $-1 \mathrm{kcal} / \mathrm{mol}$. The docking conformations of the ligands on the binding site are represented in Fig. 10a for $\beta$-Sitosterol and Fig. 10b for Calceolarioside A. The ligands were proposed to bind at Site 1 as predicted by the Qsite finder on specific residues shown in Fig. 11.

\section{Discussion}

Much attention has been given towards the screening of plant crude extracts and active constituents, but their mode of action has not been adequately discussed. The current study, therefore, attempts to delineate the role of NAT ethanolic extract in modulating the disease etiology caused by Malassezia spp. via its allergen Mala s1 through in-silico molecular simulations.
Since the advent of toxicology, MIC remains the hallmark of antimicrobial susceptibility testing but it does not guarantee success or failure of clinical trials. Therefore, in-vitro antifungal assay is now used to determine the development of antifungal susceptibility rather than the establishment of resistance [39]. This objective was materialized by turbidimetric measurements of fungal biomass for the generation of growth curves and other related parameters under the influence of increasing concentrations of the extract. The curves of extract free well demonstrate a lag phase $\left(t_{\text {lag: }}\right.$ the period until the first detectable change in OD) of $24 \mathrm{~h}$ in all species indiscriminately. Similar durations of $t_{\text {lag }}$ were observed in all tested strains indicating no inter-strain variations in growth kinetics. Moreover, it could be hypothesized that the growth patterns in different Malassezia spp. are regulated by a definite cluster of gene/proteins. When growth curves in the presence of increasing extract concentrations were studied, growth parameters were found to be affected as compared to extract free well. It could be documented that higher is the drug concentra-


Chang et al. [40], while studying the growth curves based on capacitance in Candida. The presence of drugs decreased the germination and elongation rates of spores and germinated spores, respectively, and therefore, the critical turbidity was reached later, resulting in longer lag phases.

Since fungal growth kinetics involve long incubation times, it becomes difficult to estimate the changes in OD from the start to end point. Therefore, a calculation model based on AUGC has been developed to study even small changes of the slope, which might be an indicator of transition between phases. The log phase $\left(t_{\log }\right)$ of the growth curve is that point of the curve where 


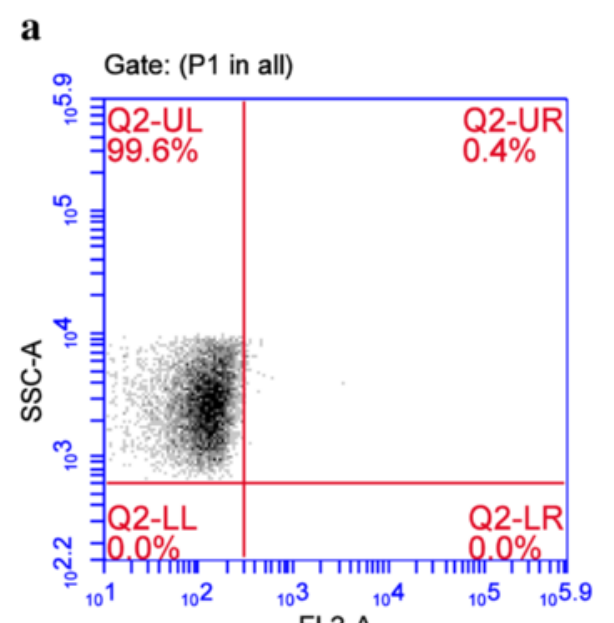

c


b

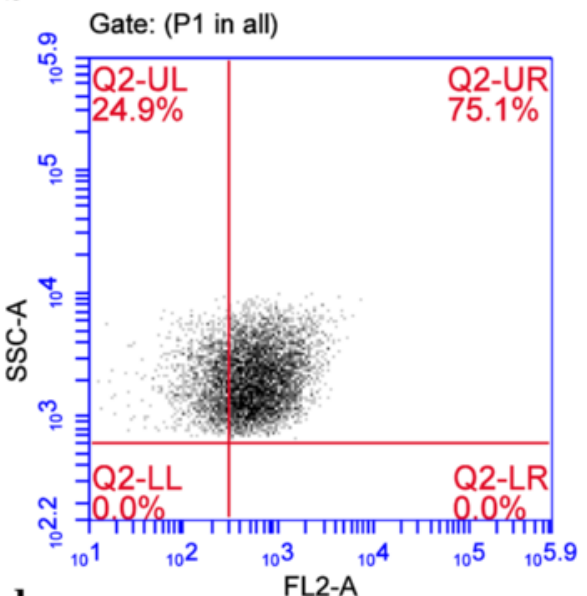

d

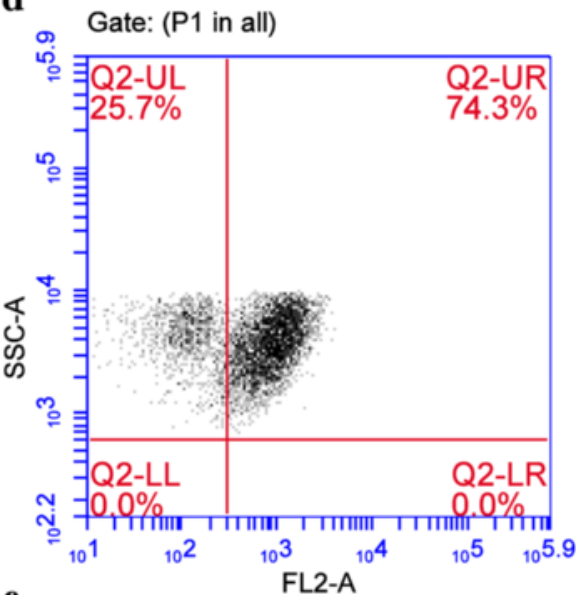

f

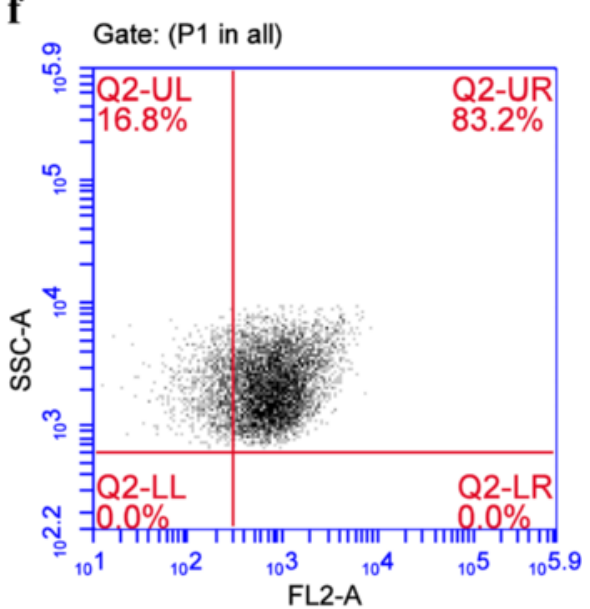

Fig. 7 Sequence density plots showing efficacy of active constituents of NAT leaf extract on lesion of plasma membrane of Malassezia sympodialis with respective percentages of PI-stained cells. a Untreated control cells, b Cells treated NAT extract at MIC $1.35 \mu \mathrm{g} / \mu \mathrm{l}$, c Cells treated with $\beta$-Sitosterol at $0.31 \mu \mathrm{g} / \mu \mathrm{l}$, d Cells treated with $\beta$-Sitosterol at $0.62 \mu \mathrm{g} / \mu \mathrm{l}$, e Cells treated with Calceolarioside A at $0.31 \mu \mathrm{g} / \mu \mathrm{l}, \mathbf{f}$ Cells treated with Calceolarioside A at $0.62 \mu \mathrm{g} / \mu \mathrm{l}$

AUGC values increase linearly over time and the growth rate is constant at its highest value [41, 42]. AUGC represented a strong correlation with the extract concentrations $\left(R^{2}=0.837\right)$ in $M$. furfur with about $50 \%$ decrease in AUGC under highest dose effect as compared to extract free well. Other species expressed results in concordance with $M$. furfur, indicating that the dose range selected was equally effective over other species 


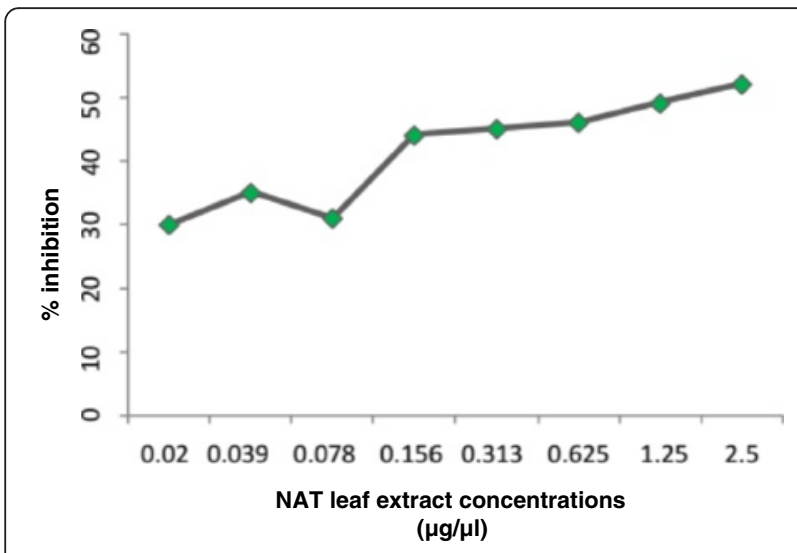

Fig. 8 Antioxidant activity of NAT leaf extract of various dose ranges $(0.02-2.5 \mu \mathrm{g} / \mu \mathrm{l})$ was treated with DPPH cation to obtain FRSA spectrophotometrically

under consideration and their growth kinetics in $t_{\log }$ was also in proximity.

For further determination of quality control of NAT extract, their MIC range in tested strains was established. This was accomplished using sensitive and reproducible spectrophotometrically system rather that manual visualization. The MIC for $M$. furfur, $M$. restricta, M. globosa, and M. sympodialis extended from 1.05 to $1.47 \mu \mathrm{g} / \mu \mathrm{l}$. Percent growth inhibition curves indicated high correlation values with concentrations. A linear regression attributed towards strong dose dependent growth in all fungal strains. Holovachuk et al. [43] reported an inverse relationship between concentration of Gentamicin (log transformed) and bacterial growth based on AUGC. The equation used for the line was $\mathrm{Y}=\mathrm{A}+\mathrm{B} \times \log (\mathrm{X})$, where $\mathrm{Y}=\mathrm{AUGC}$, $\mathrm{A}$ is the $\mathrm{y}$ intercept, $B$ is the slope of log transformed data and $X$ is the drug concentration. With reference to the above analysis, we established a linear relationship between fungal growth based AUGC and extract concentration (log transformed). Over the dose range of NAT extract
Table 2 Protein-ligand docking results from HEX server, docked energies obtained from protein-ligand docking of Mala s1 allergen of Malassezia spp. with $\beta$-Sitosterol

\begin{tabular}{lllll}
\hline Receptor protein & Ligand & Cluster & Solution & Etotal \\
\hline Mala s1 & B-Sitosterol & 1 & 1 & -283.1 \\
& 2 & 1 & -239.7 \\
& 3 & 1 & -229.8 \\
& 4 & 1 & -223.6 \\
& 5 & 1 & -219.7 \\
6 & 1 & -216.1 \\
& 7 & 1 & -213.2 \\
& 8 & 1 & -210.7 \\
& 9 & 1 & -208.4 \\
& 10 & 1 & -206.1 \\
\hline
\end{tabular}

from 0.02 to $2.5 \mu \mathrm{g} / \mu \mathrm{l}$ there existed a direct relationship between drug concentration and the growth phase. Lower extract concentration did not inhibit growth. Plotting AUGC against extract concentrations (log transformed) yielded similar findings indicating comparable working concentration range of the assay for all test pathogens.

During infection, skin bears a significant fungal load but with a distinct absence of inflammation. This down regulation of host inflammatory cascade may be attributed to the production of the fungal metabolites [44]. In such conditions the isolation and identification of the causal organisms and their molecular validation becomes an important tool for the establishment of pathogenic conditions of PV. Down regulation in band intensities of extract treated Malassezia cultures as compared to control indicated nucleic acid damage thereby depleting fungal population.

Earlier studies have shown that primary mechanism of action of various antifungal agents is through the disruption of plasma membranes and interfering with the
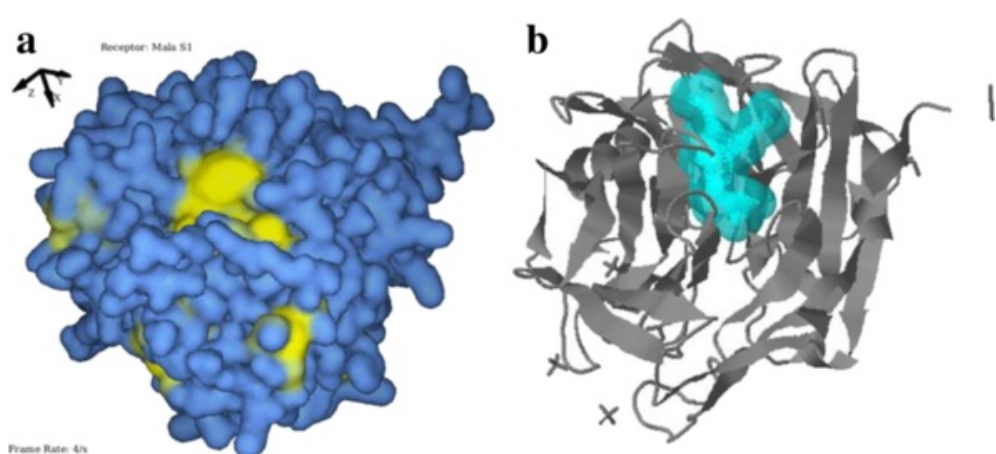

Fig. 9 Structure and Binding sites prediction of of Mala s1. a The binding sites are represented in yellow color over the blue background of solid surface model of Mala s1 allergen and $\mathbf{b}$ exhibit specific binding site (blue) of the natural ligand in cartoon representation of Mala s1 allergen (grey). The sites are analyzed using Q-Site finder server predictions 
Table 3 Protein-ligand docking results from HEX server, docked energies obtained from protein-ligand docking of Mala s1 allergen of Malassezia spp. with Calceolarioside A

\begin{tabular}{lllll}
\hline Receptor protein & Ligand & Cluster & Solution & Etotal \\
\hline Mala s1 & Cal A & 1 & 1 & -354.9 \\
& 2 & 1 & -263.3 \\
& 3 & 1 & -252.5 \\
& 4 & 1 & -245.0 \\
& 5 & 1 & -240.1 \\
& 6 & 1 & -236.1 \\
& 7 & 1 & -232.5 \\
& 8 & 1 & -229.5 \\
& 9 & 1 & -227.0 \\
& 10 & 1 & -224.7 \\
\hline
\end{tabular}

normal biosynthetic pathways [45]. This attribute was studied in terms of PI influx in fungal cells treated with leaf extract as well as with two active constituents of NAT by flow cytometry. PI is a membrane-impermeabale fluorescent stain that penetrates damaged or permeabilized plasma membrane and binds to nucleic acid [46]. The whole plant extract of NAT has been found to disrupt plasma membrane and facilitate the permeabilization of PI. Also similar observations have been made with Calceolarioside $\mathrm{B}$ and $\beta$-Sitosterol. These results indicate that active constituents alter the cell membrane structure, causing disruption of membrane permeability barrier.

The skin diseases cause disruption of the adjoining cells and clinically results in skin fragility and blistering. Therefore, restoring and rejuvenating the skin components becomes an important aspect along with the treatment of pathogens. In this aspect antioxidants play a vital role and constitute an integral component of

\section{$\mathbf{a}$}
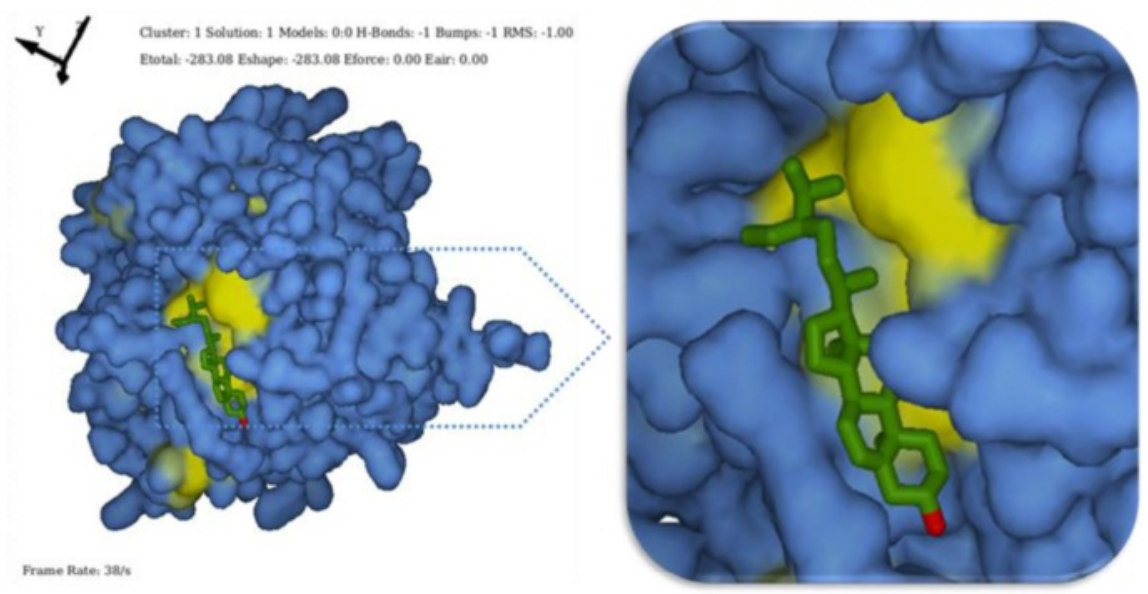

b

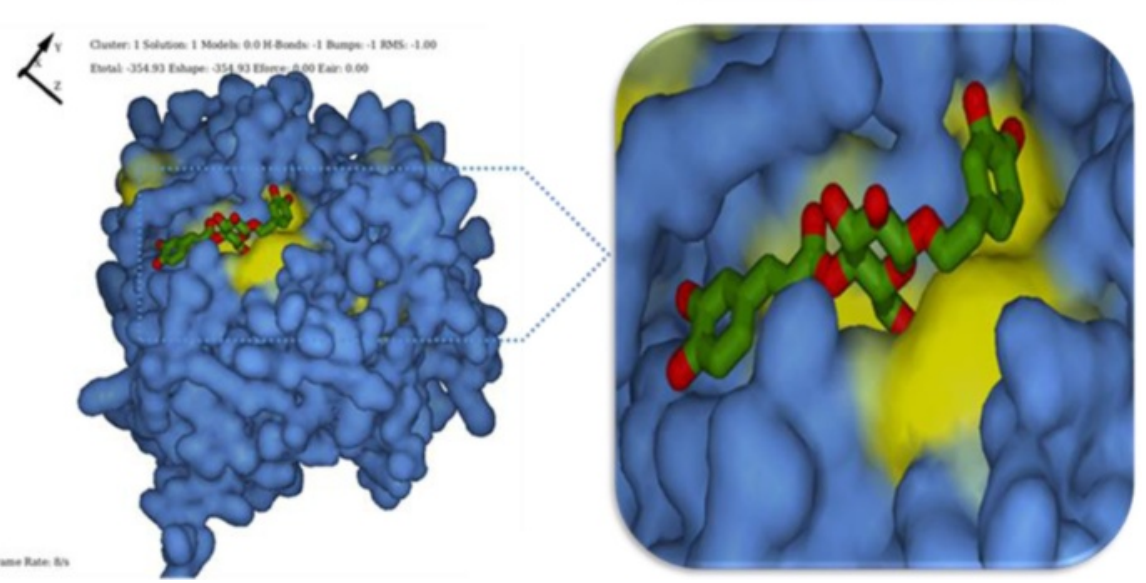

Fig. 10 Protein-ligand docking conformation. The Protein Mala s1 allergen of Malassezia sympodialis is represented in blue solid surface model with yellow binding pockets. a The ligand $\beta$-Sitosterol represented in licorice form is embedded in the binding pocket. The binding mode as predicted by the docking program HEX (6.12v) gives an Etotal of -283.03 (left). The docked sites are also represented as an enlarged view (right). b The ligand Calseolarioside A represented in licorice form is embedded in the binding pocket. The binding mode as predicted by the docking program HEX (6.12v) gives an Etotal of -354.93 (right). The docked sites are also represented as an enlarged view (right) 



Fig. 11 Detailed representations of binding residues of Mala s1 allergen with $\mathbf{a} \beta$-Sitosterol and $\mathbf{b}$ Calceolarioside A. Mala s1 is represented in chain form with binding sites in mesh form (green). The residues were identified using Atom Picking mode of docking software HEX

topical ointments. Recent studies have shown that the leaves and stem of Nyctanthes arbor-tristis are a potential source of natural antioxidants [47]. Ethanolic extracts of leaves and stem contains secondary metabolites of several classes viz., flavonoids, tannins, saponins, glycosides, alkaloids, steroids and phenolic compounds, of which phenolic compounds are also responsible for its antioxidant activity $[48,49]$. The antioxidant activity in form of percent inhibition of DPPH radical showed a uniform increase with the increase in the concentration of the extract. Since inflammation and oxidative stress appear to play a significant role in Pityriasis versicolor, leaves of NAT may play an important role in wound healing through its antioxidant capacity facilitating free radicals scavenging thereby down regulating skin inflammation. Thus, the antioxidant potential marks an added advantage in the treatment of skin inflammation.

Mechanisms of action studies of natural molecules are fundamental to drug discovery. However, in-vitro studies alone may not recapitulate compound's MoA in whole cell. Therefore, with the establishment of anti-Malassezia capacity of NAT in-vitro, we employed in-silico modeling of Mala s1 allergen of $M$. sympodialis with the bioactive constituents from NAT. Genes encoding for all 10 allergens previously cloned in different species of Malassezia, are now reported to exist in M. sympodialis genome [50]. Thus, in the current study, we addressed Mala s1 allergen of $M$. sympodialis (PDB ID: 2P9W) as a model to advance our understanding of how the yeasts interact with the host and contributes to disease pathogenesis. The localization of Mala s1 on the cell surface [51] and its role in disease etiology merits the background and frame of further investigations. Further, docking was performed between protein and active molecules ( $\beta$-Sitosterol and
Calceolarioside A) using Hex server and their interaction energies were analyzed (Tables 2 and 3). The low energies ensured efficient docking and also revealed that the protein could form hydrogen bond networks involving various active amino acid residues [52]. Figure 11 reveals various residues that exclusively contribute towards the binding of the protein with the natural molecules. Cumulatively, the evidence leads us to propose that the potent inhibition of cell surface allergen Mala s1 by the active constituents of NAT makes the later a unique and useful tool among the pre-existing synthetic antifungals. However, it is tempting to validate the sub-cellular machinery that will further enlighten the stage to identify new targets and their cognate inhibitors for development of novel antifungal therapeutics.

\section{Conclusion}

The findings stated above provide an insight about the medicinal impact of NAT leaf ethanolic extract that has an inhibitory effect over Malassezia spp. The growth curves and the related parameters indicate that the extract can be used against different strains of Malassezia spp. The fungal DNA depletion associated with extract treatment also signifies the therapeutic potential of the extract. The disruption of cell membrane as observed by flow cytometry further confirms the role of active constituents as antifungal agents by driving membraneactive mechanism. Moreover, in-silico prediction identified specific binding sites on Mala s1 allergen that could be useful for structure-based drug designing. Further, the virtual screening of two active constituents from NAT leaf extract and their efficient docking with Mala s1 open up new avenues for the development of plant based antifungal formulations for skin care after 
generating toxicological data as well as successful topical testing.

\section{Availability of data and materials}

The chemical structures of the active constituents were obtained from http://pubchem.ncbi.nlm.nih.gov/. The crystal structure of protein was obtained from http:// www.rcsb.org.

\author{
Abbreviations \\ AUGC: Area under growth curve; DPPH: Diphenylpicryl-hydrazyl; \\ MFCs: Minimum fungicidal concentrations; MICs: Minimum inhibitory \\ concentrations; NAT: Nyctanthes arbor-tristis L.; PI: Propidium lodide; \\ SE: Standard error
}

\section{Competing interests}

The authors declare that they have no competing interests.

\section{Authors' contributions}

RKM and VM performed the turbidimetric growth assay, flow cytometery and in silico simulations. VM and SS drafted the manuscript. AP carried out the antioxidant assays of NAT extract. HP and AT prepared the extract. AD and ACP designed the study. All authors read and approved the final manuscript.

\section{Acknowledgements}

This work was supported by Young Scientist grant (SB/YS/LS-44/2014) funded by Science and Engineering Research Board (SERB), Department of Science and Technology (DST), Dr. D. S. Kothari Post Doctoral Fellow grant (F.4-2/2006 (BSR)/13-813/2013 (BSR) by University Grant Commission (UGC), New Delhi, India and Design and Innovation Centre (DIC), Ministry of Human Resource and Development (MHRD), New Delhi. Authors are thankful to Prof. Hem Lata Joshi for critically reviewing the manuscript.

\section{Author details}

${ }^{1}$ Centre for Medical Diagnostic and Research (CMDR), Motilal Nehru National Institute of Technology (MNNIT), Allahabad 211004, India. ${ }^{2}$ Nanotechnology Application Centre (NAC), University of Allahabad, Allahabad 211002, India. ${ }^{3}$ Department of Botany, Biological Product Lab., University of Allahabad, Allahabad 211002, India. ${ }^{4}$ Department of Pharmaceutical Sciences, Sam Higginbottom Institute of Agriculture Technology and Sciences, Allahabad 211007, India.

Received: 2 May 2015 Accepted: 23 March 2016

Published online: 31 March 2016

\section{References}

1. Leeming JP, Notman FH, Holland KT. The distribution and ecology of Malassezia furfur and cutaneous bacteria on human skin. J Appl Bacteriol. 1989:67:47-52.

2. Soares RC, Zani MB, Arruda ACBB, Arruda LHFD, Paulino LC. Malassezia intra-specific diversity and potentially New species in the skin microbiota from Brazilian healthy subjects and seborrheic dermatitis patients. PLoS One. 2015;10(2):e0117921.

3. Velegraki A, Cafarchia C, Gaitanis G, latta R, Boekhout T. Malassezia infections in humans and animals: Pathophysiology, detection, and treatment. PLoS Pathog. 2015;11(1):e1004523.

4. Leung DY, Boguniewicz M, Howell MD, Nomura I, Hamid QA. New insights into atopic dermatitis. J Clin Invest. 2004;113:651-7.

5. Gupta AK, Bluhm R, Summerbell R. Pityriasis versicolor. J Eur Acad Dermatol Venereol. 2002;16:19-33.

6. Tarazooie B, Kordbacheh P, Zaini F, Zomorodian K, Saadat F, Zeraati H, et al. Study of the distribution of Malassezia species in patients with pityriasis versicolor and healthy individuals in Tehran, Iran. BMC Dermatol. 2004;4:5. doi:10.1186/1471-5945-4-5

7. Gaitanis G, Magiatis P, Hantschke M, Bassukas ID, Velegraki A. The Malassezia genus in skin and systemic diseases. Clin Microbiol Rev. 2012;25:106-41.

8. Plato A, Hardison SE, Brown GD. Pattern recognition receptors in antifungal immunity. Semin Immunopathol. 2015;37:97-106.
9. Lintu P, Savolainen J, Kalimo K. IgE antibodies to protein and mannam antigens of pityrosporum ovale in atopic dermatis patients. Clin Exp Allergy. 1997;27:87-95

10. Zargari A. Identification and characterization of allergen components of the opportunistic Malassezia furfur yeast. Stockholm. 1998;50-56. http://hdl. handle.net/10616/43923. ISBN: 91-628-2997-1.

11. Zargari A, Eshaghi H, Back O, Johansson S, Scheynius A. Serum IgE reactivity to Malassezia furfur extract and recombinant M. furfur allergens in patients with atopic dermatitis. Acta Derm Venereol. 2001;81:418-22.

12. Hammer KA, Carson CF, Riley TV. In-vitro activities of ketoconazole, econazole, miconazole, and melaleuca alternifolia (Tea tree) oil against Malassezia species. Antimicrob Agents Chemther. 2000;44:467-9.

13. Jagielski T, Rup E, Ziółkowska A, Roeske K, Macura AB, Bielecki J. Distribution of Malassezia species on the skin of patients with atopic dermatitis, psoriasis, and healthy volunteers assessed by conventional and molecular identification methods. BMC Dermatol. 2014;14(3):1-15.

14. Watanabe S, Koike A, Kano R, Nagata M, Chen C, Hwang CY, et al. In-vitro Susceptibility of Malassezia pachydermatis Isolates from Canine Skin with Atopic Dermatitis to Ketoconazole and Itraconazole in East Asia. J Vet Med Sci. 2014;76(4):579-81.

15. Fera MT, Camera ELC, De Sarro A. New triazoles and echinocandins: mode of action, in vitro activity and mechanisms of resistance. Expert Rev Anti Infect Ther. 2009;7:981-98.

16. Jesus FPK, Lautert C, Zanette RA, Mahl DL, Azevedo MI, Machado MLS, et al. In-vitro susceptibility of fluconazole-susceptible and resistant isolates of Malassezia pachydermatis against azoles. Vet Microbiol. 2011;152:161-4.

17. Ji HF, Li XJ, Zhang HY. Natural products and drug discovery. EMBO Rep. 2009:10:194-200.

18. Astin J. Why patients use alternative medicine: results of a national study. JAMA. 1998:279:1548-53.

19. Pandey A, Mishra RK, Tiwari AK, Kumar A, Bajaj AK, Dikshit A. Management of cosmetic embarrassment caused by Malassezia spp. With fruticose lichen cladia using phylogenetic approach. BioMed Res Int. 2013;2013:169794.

20. Kumari P, Sahal D, Jain SK, Chauhan VS. Bioactivity Guided Fractionation of Leaves Extract of Nyctanthes arbortristis (Harshringar) against $P$. falciparum. PLoS One. 2012:7(12):e51714. doi:10.1371/journal.pone.0051714.

21. Saxena RS, Gupta B, Saxena KK, Srivastava VK, Prasad DN. Analgesic, antipyretic and ulcerogenic activity of Nyctanthes arbor tristis leaf extracts. J Ethnopharmacol. 1987;19:193-200.

22. Verma N, Kaur J, Bhatia A. Stimulation of acetylcholinesterase activity with Nyctanthes arbor-tristis leaves extract in the malathion-treated immunosuppressed mice. Int J Environ Stud. 2001;58:645-54

23. Rathore B, Paul B, Chaudhury BP, Saxena AK, Sahu AP, Gupta YK. Comparative studies of different organs of Nyctanthes arbortristis in modulation of cytokines in murine model of arthritis. Biomed Environ Sci. 2007:20:154-9

24. Khan ZK, Manglani A, Shukla PK, Puri A, Saxena RP, Tandon JS. Immunomodulatory effect of plant extracts and iridoid glucosides from Nyctanthes arbortristis against systemic candidiasis in mice. Int J Pharmacogn. 1995;33:297-304.

25. Agarwal J, Pal A. Nyctanthes arbor-tristis Linn. A critical ethnopharmacological review. J Ethanopharmacol. 2013;146(3):645-58. doi:10.1016/j.jep.2013.01.024.

26. Alvarez-Perez S, Blanco JL, Pelaez T, Cutuli M, Garcíaa ME. In-vitro Amphotericin B Susceptibility of Malassezia pachydermatis determined by the CLSI Broth Microdilution Method and Etest Using Lipid-Enriched Media. Antimicrob Agents Chemther. 2014;58(7):4203-6.

27. Baroni A, Perfetto B, Paoletti I, Ruocco E, Canozo N, Orlando M, et al. Malassezia furfur invasiveness in a keratinocyte cell line (HaCat): effects on cytoskeleton and on adhesion molecule and cytokine expression. Arch Dermatol Res. 2001;293:414-9.

28. Werner S, Krieg T, Smola H. Keratinocyte-fibroblast in wound healing. I Invest Dermatol. 2007;127:998-1008.

29. Saha RK, Acharya S, Shovon S, Apu AS, Roy P. Biochemical investigation and biological evaluation of the methanolic extract of the leaves of Nyctanthes arbortristis in-vitro. Asian Pacific J Trop Biomed. 2012 doi:10.1016/S2221-1691(12)60449-3.

30. Meghashri S, Gopal S. Biochemical characterization of radical scavenging polyphenols from Nyctanthes arbortristis. J Pharm Bioallied Sci. 2012:4:341-4.

31. Clinical and Laboratory Standards Institute. Reference method for broth dilution antifungal susceptibility testing of yeasts; approved standard. 3rd ed. CLSI document M27-A3 2008, Wayne PA. 
32. Clinical and Laboratory Standards Institute. Performance Standards for Antimicrobial Disk Susceptibility Tests; Approved Standard-Eleventh Edition. M02-A11 2012, Wayne, PA.

33. Dikshit A, Tiwari AK, Mishra RK. A culture medium for the growth of Malassezia spp. Technology Information, Forecasting and Assessment Council (TIFAC), Department of Science and Technology (DST), New Delhi, Filed for patent App. No. 546/DEL/2012, 2012.

34. Dikshit A, Tiwari AK, Mishra RK. New medium for rapid diagnosis and determination of antifungal testing against Malassezia spp.: A Potential Candidate for Industries. Natl Acad Sci Lett. 2013. doi:10.1007/s40009-012-0081-3.

35. Brand-Williams W, Cuvelier ME, Berset C. Use of a free radical method to evaluate antioxidant activity. Leb Wiss Technol. 1995;28:25-30.

36. Laurie ATR, Jackson RM. Q-SiteFinder: an energy-based method for the prediction of protein-ligand binding sites. Bioinform. 2005;21:1908-16.

37. Vilhelmsson M, Zargari A, Crameri R, Rasool O, Achour A, Scheynius A, et al. Crystal structure of the major Malassezia sympodialis allergen Mala s1 reveals a $\beta$-propeller fold: A novel fold among allergens. J Mol Biol. 2007;369:1079-86

38. Ritchie DW, Kozakov D, Vajda S. Accelerating and focusing proteinproteindocking correlations using multi-dimensional rotational FFT generating functions. Bioinformatics. 2008;24:1865-73.

39. Kontoyiannis DP, Lewis RE. Antifungal drug resistance of pathogenic fungi. Lancet. 2002;359:1135-44.

40. Chang HC, Chang JJ, Huang AH, Chang TC. Evaluation of a capacitance method for direct antifungal susceptibility testing of yeasts in positive blood cultures. J Clin Microbiol. 2000;38:971-6.

41. Meletiadis J, Meis JF, Mouton JW, Verweij PE. Analysis of growth characteristics of filamentous fungi in different nutrient media. J Clin Microbiol. 2001;39:478-84.

42. Meletiadis J, te Dorsthorst DTA, Verweij PE. Use of turbidimetric growth curves for early determination of antifungal drug resistance of filamentous fungi. J Clin Microbiol. 2003;41:4718-25.

43. Holowachuk SA, Bal MF, Buddington RK. A kinetic microplate method for quantifying the antibacterial properties of biological fluids. J Microbiol Meth. 2003:55:441-6.

44. Mayser P, Gaitanis G. Physiology and biochemistry. In: Boekhout T, Gueho E, Mayser P, Velegraki A, editors. Science and clinical practice. Germany: Springer; 2010. p. 121-38.

45. Kelly SL, Lamb DC, Corran AJ, Baldwin BC, Kelly DE. Mode of action and resistance to azole antifungals associated with the formation of 14 alphamethylergosta-8,24(28)-dien-3 beta,6 alpha-diol. Biochem Biophys Res Commun. 1995:207:910-5.

46. Ali I, Khan FG, Suri KA, Gupta BD, Satti NK, Dutt P, et al. In-vitro antifungal activity of hydroxychavicol isolated from Piper beetle L. Ann Clin Microbiol Antimicrob. 2010;9:7

47. Thangavelu R, Thomas S. In-vitro antioxidant studies on ethanolic extract of leaves and stems of Nyctanthes arbor-tristis L. (Night flowering Jasmine). Int J Biol Med Res. 2010;1(4):188-92.

48. Devasagayam TPA, Sainis KB. Immune system and antioxidants, especially those derived from Indian medicinal plants. Indian J Exp Biol. 2002:40:639-55.

49. Rani C, Chawla S, Mangal M, Mangal AK, Kajla S, Dhawan AK. Nyctanthes arbor-tristis Lnn. (Night Jasmine): A sacred ornamental plant with immense medicinal potentials. Ind J Trad Knowledge. 2012;11(3):427-35.

50. Gioti A, Nystedt B, Li W, Xu J, Andersson A, Averettee AF, et al. Genomic insights into the atopic eczema-associated skin commensal yeast Malassezia sympodialis. Mbio. 2013;4(1):e00572-12. doi:10.1128/mBio.00572-12.

51. Zargari A, Emilson A, Hallden G, Johansson S, Scheynius A. Cell surface expression of two major yeast allergens in the Pityrosporum genus. Clin Exp Allergy. 1997;27:584-92.

52. Kumar GR, Chikati R, Pandrangi SL, Kandapal M, Sonkar K, Gupta N, et al. Molecular docking and dynamics simulations of A. niger RNase from Aspergillus niger ATCC-26550: for potential prevention of human cancer. J Mol Model. 2013;19:613-21. 